\title{
Evaluation of Kidney Function Tests in HIV-Positive Patients Receiving Combined Antiretroviral Therapy
}

\author{
emre aydın ${ }^{1}$ Fatma Yılmaz Aydın¹, Yakup Demir ${ }^{1}$, Yaşar Yıldırım , and Mustafa Kemal \\ Çelen ${ }^{1}$ \\ ${ }^{1}$ Dicle University Medical Faculty
}

April 10, 2021

\begin{abstract}
Introduction: Human Immunodeficiency virus is a chronic infection that attacks the immune system of the human body, particularly CD4 T lymphocytes. Combined antiretroviral therapies are highly effective in virological suppression of human immunodeficiency virus infection. It has been shown that some retroviral therapies have a higher nephrotoxicity potential. As a result of renal injury, serum creatinine increases, and the estimated glomerular filtration rate is reduced. The aim of our study was to assess changes in kidney function during a 24-month period in HIV-positive patients who were begun on combined antiretroviral therapy. Material-method: A total of 127 HIV positive patients were enrolled. The patients were divided into five groups; patients who received no therapy were designated as Group 1; those that received Dolutegravir/Abacavir/Lamivudine combination as Group 2; those that received Elvitegravir/Cobicistat/Emtricitabine/Tenofovir Alafenamide Fumarate combination as Group 3; those that received Emtricitabine/Tenofovir Disoproxil Fumarate/Dolutegravir combination as Group 4; and those that received Emtricitabine/Tenofovir Disoproxil Fumarate/Raltegravir combination as Group 5. We compared the effects of these drugs on estimated glomerular filtration rate during a 24-month follow-up period. Results: At the 24th month of therapy, a significant difference was observed between the eGFR levels of the study groups ( $\mathrm{p}:<0.001$ ). eGFR level was significantly higher in Group 4 compared to Groups 1, 2, and 3 (p:0.009, p: $<0.001, \mathrm{p}:<0.001$, respectively) while it was significantly lower in Group 5 than groups 1, 2, and 3 (p:0.005, p: $<0.001, \mathrm{p}:<0.001$, respectively). No significant eGFR difference was found between Group 4 and Group 5 ( $>>0.05$ ). Serum creatinine level was significantly higher in Groups 4 and 5 compared to the other groups $(\mathrm{p}<0.001)$. Conclusion: The use of TDF-containing regimens causes renal dysfunction. Therefore, we recommend close monitoring of renal function, especially in patients treated with TDF.
\end{abstract}

\section{Evaluation of Kidney Function Tests in HIV-Positive Patients Receiving Combined Antiretro- viral Therapy}

\section{Antiretroviral Therapy and Kidney}

AbstractIntroduction: Human Immunodeficiency virus is a chronic infection that attacks the immune system of the human body, particularly CD4 T lymphocytes. Combined antiretroviral therapies are highly effective in virological suppression of human immunodeficiency virus infection. It has been shown that some retroviral therapies have a higher nephrotoxicity potential. As a result of renal injury, serum creatinine increases, and the estimated glomerular filtration rate is reduced. The aim of our study was to assess changes in kidney function during a 24-month period in HIV-positive patients who were begun on combined antiretroviral therapy.Material-method: A total of $127 \mathrm{HIV}$ positive patients were enrolled. The patients were divided into five groups; patients who received no therapy were designated as Group 1; those that received Dolutegravir/Abacavir/Lamivudine combination as Group 2; those that received Elvitegravir/Cobicistat/Emtricitabine/Tenofovir Alafenamide Fumarate combination as Group 3; those that received Emtricitabine/Tenofovir Disoproxil Fumarate/Dolutegravir combination as Group 4; and those 
that received Emtricitabine/Tenofovir Disoproxil Fumarate/Raltegravir combination as Group 5. We compared the effects of these drugs on estimated glomerular filtration rate during a 24-month follow-up period.Results: At the 24th month of therapy, a significant difference was observed between the eGFR levels of the study groups ( $\mathrm{p}:<0.001)$. eGFR level was significantly higher in Group 4 compared to Groups 1,2 , and 3 (p:0.009, p: $<0.001, \mathrm{p}:<0.001$, respectively) while it was significantly lower in Group 5 than groups 1, 2, and 3 (p:0.005, p: $<0.001, \mathrm{p}:<0.001$, respectively). No significant eGFR difference was found between Group 4 and Group 5 ( $\mathrm{p}>0.05)$. Serum creatinine level was significantly higher in Groups 4 and 5 compared to the other groups $(\mathrm{p}<0.001)$.Conclusion: The use of TDF-containing regimens causes renal dysfunction. Therefore, we recommend close monitoring of renal function, especially in patients treated with TDF.Keywords: Human Immunodeficiency Virus, Nephrotoxicity, Tenofovir, estimated glomerular filtration rate

\section{What's already known about this topic?}

cARTs are known to exert beneficial effects on the natural course of the HIV infection and patient survival. However, some antiretroviral drugs have nephrotoxic side effects. As a result of the developing nephrotoxicity, the risk of CKD increases.

\section{What does this article add?}

There is no study evaluating all treatment protocols in terms of nephrotoxicity in this period when new cARTs options are on the agenda. In addition, since this study is the first study in our geography, we believe that it will guide the treatment options in this patient population.

\section{Introduction}

Human Immunodeficiency Virus (HIV) is a chronic infection attacking the immune system of the human body, particularly targeting CD4 lymphocytes. It may cause fatal consequences by leading to the Acquired Immune Deficiency Syndrome (AIDS), which is characterized by opportunistic infections as a result of immune suppression. HIV-infected persons should be begun on antiretroviral therapy (ART) as soon as possible, both to improve their own survival rate and to reduce the risk of transmitting the disease to others $^{1}$. ARTs are known to exert beneficial effects on the natural course of the HIV infection and patient survival ${ }^{2}$. Combined ARTs (cARTs) are highly effective in the virological suppression of HIV infection ${ }^{3}$. In addition to two nucleoside analog reverse transcriptase inhibitors (NRTIs), the combined use of a third active drug is recommended, namely an integrase strand transfer inhibitor (INSTI), a non-nucleoside reverse transcriptase inhibitor (NNRTI), or an amplified protease inhibitor (PI) for the treatment of HIV-infected persons ${ }^{4}$. HIV-positive patients are at risk of both acute kidney injury (AKI) and chronic kidney disease (CKD) due to drug nephrotoxicity, HIV-associated nephropathy (HIVAN), and immune complex kidney diseases $(\mathrm{HIVICK})^{5}$. Studies have shown that some ARTs have a greater nephrotoxicity potential ${ }^{6-8}$. While these drugs can cause direct kidney injury by tubular dysfunction, acute interstitial nephritis, and kidney stones, they also cause kidney injury via drug-drug interactions, drug dosing errors, and ART-induced rhabdomyolysis, lactic acidosis, and metabolic complications ${ }^{9}$. Kidney injury results in increased serum creatinine and reduced estimated glomerular filtration rate (eGFR). ART-associated kidney injury is an important cause of mortality and morbidity. Hence, it is important to monitor serum creatinine and eGFR during ART. The aim of our study was to assess the changes in renal function during a 24-month period in HIV-positive patients who received cART.Material-methods The medical records of $140 \mathrm{HIV}$-positive patients who were followed and treated between 2017 and 2019 were retrospectively reviewed. HIV-positive patients older than 18 years were enrolled irrespective of sex whereas patients younger than 18 years, those who had used antiviral therapy, those who were receiving nephrotoxic agents, and those who had a baseline eGFR level below $60 \mathrm{~mL} / \mathrm{min} / 1.73 \mathrm{~m} 2$ were excluded. As a result, 127 patients were enrolled per the study protocol (Figure 1). The study was approved by the Dicle University Faculty of Medicine local ethics committee (06.02.2020/146). The patients were divided into 5 groups: Group $1(\mathrm{n}=11)$ : Untreated HIV-positive patients. Group $2(\mathrm{n}=19)$ : HIV-positive patients treated with Dolutegravir (DTG) + Abacavir (ABC) + Lamivudine (3TC) combination. Group $3(\mathrm{n}=31)$ : HIV-positive patients treated with Elvitegravir (EVG)/ Cobicistat (COBI) + Emtricitabine (FTC) + Tenofovir Alafenamide Fumarate (TAF) combination. Group 4 
$(\mathrm{n}=33)$ : HIV-positive patients treated with Emtricitabine (FTC) + Tenofovir Disoproxil Fumarate (TDF) + Dolutegravir (DTG) combination. Group 5 (n=33): HIV-positive patients treated with Emtricitabine (FTC) + Tenofovir Disoproxil Fumarate (TDF) + Raltegravir (RAL) combination. Age, sex, height, weight, body mass index, pre-treatment HIV RNA level, CD4, CD8 levels, as well as the pre-treatment and post-treatment 6th, 12th, and 24th-month creatinine and eGFR levels were recorded. eGFR level was calculated with the 4variable Modification of Diet in Renal Disease (MDRD) formula. Then, the eGFR levels of the study groups were compared.Statistical AnalysisStudy data were statistically analyzed using SPSS (Statistical Package for Social Sciences) version 24.0 software package (SPSS Inc, Chicago, IL). The normality of the distribution of the study variables was tested with visual (histogram and likelihood graphics) and analytic methods (Kolmogorov - Smirnov / Shapiro - Wilk tests). The results were reported as number and percentage for categoric variables and mean \pm standard deviation for continuous variables. The effects of treatment and time on eGFR were evaluated using repeated ANOVA measurements. Mauchly's Test of Sphericity was used to check the assumption of sphericity. If Mauchly's test statistic was significant, the Greenhouse - Geisser or Huynh - Feldt correction was used. If the main/interaction effect was significant, a Bonferroni correction was applied for multiple comparisons. According to our treatment groups, the values of eGFR over time are presented with a profile plot (Figure 2). Inter-group analysis of non-normally distributed variables was performed with Kruskal - Wallis test, with Mann - Whitney U test being used for paired group comparisons. A p-value of less than 0.05 was considered statistically significant.Results The 24-month follow-up data of 127 patients were reviewed and the groups were compared (Table 1). One hundred and seven (84.3\%) patients were male, and $20(15.7 \%)$ patients were female. The mean age was $29.37 \pm 7.06$ years, and the mean BMI was $27.45 \pm 3.65$. While there was no significant difference between the study groups with respect to age, sex, BMI, pre-treatment CD8 level, creatinine, and eGFR levels, significant differences were detected regarding pre-treatment HIV-RNA, CD4 level, CD4/CD8 ratio (p: $<0.001$ for all comparisons). Pre-treatment CD4 level and the CD4/CD8 ratio were significantly lower whereas HIV-RNA level was significantly higher in Groups 2, 3, 4, and 5 (p: $<0.001$, for all comparisons). Groups 3 and 4 had significantly higher CD4 levels than Group $2(\mathrm{p}<0.05)$. Groups 3, 4, and 5 had significantly lower HIV-RNA levels than Group $2(\mathrm{p}<0.05)$. The groups' pre-treatment, 6 th month, 12 th month, and 24th-month serum creatinine and eGFR levels were calculated and compared. eGFR levels at the 6 th month of therapy were significantly different between the study groups (p:0.002). Inter-group comparison of eGFR level showed that it was significantly lower in Group 4 compared with Groups 1, 2, and 3 (p:0.023, p:0.033, p:0.001, respectively), and also significantly lower in Group 5 than Group 1 and 3 (p:0.029, p:0.003, respectively). However, there was no significant difference between Group 4 and Group 5 ( $>>0.05$ ). Serum creatinine level was significantly higher in Groups 4 and 5 than in the other groups $(\mathrm{p}<0.05)$. An analysis of eGFR levels at the 12 th month of therapy showed a significant difference between the groups $(\mathrm{p}:<0.001)$. Group 4 had a significantly lower eGFR level than Groups 1, 2, and 3 (p:0.002, p: $<0.001, \mathrm{p}: 0.001$, respectively); similarly, Group 5 had a significantly lower eGFR level compared with Groups 1, 2, and 3 (p:0.002, p: $<0.001, \mathrm{p}: 0.001$, respectively). On the other hand, Group 4 and Group 5 showed no significant difference $(\mathrm{p}>0.05)$. Serum creatinine level was significantly higher in Groups 4 and 5 compared with the other groups $(\mathrm{p}<0.001)$. The study groups showed significant differences regarding the 24 th-month eGFR levels $(\mathrm{p}:<0.001)$. Group 4 had a significantly lower eGFR than Groups 1, 2, and 3 (p:0.009, p: $<0.001, \mathrm{p}:<0.001$, respectively); similarly, Group 5 had a significantly lower eGFR level than Groups 1, 2, and 3 (p:0.005, p: $<0.001, \mathrm{p}:<0.001$, respectively). However, no significant difference was found between Group 4 and Group $5(\mathrm{p}>0.05)$. Serum creatinine level was significantly greater in Groups 4 and 5 than in the other groups $(\mathrm{p}<0.001)$. Figure 2 shows monthly eGFR changes within treatment groups and inter-group eGFR comparisons. Temporal eGFR change was not statistically significant in Groups 1, 2, and 3 (p:0.397, p:0.448, p:0.886, respectively). A significant decrease in eGFR was observed in Groups 4 and 5 at 6 th month (p: $<0.001), 12$ th month $(\mathrm{p}:<0.001)$, and 24 th month $(\mathrm{p}:<0.001)$ compared to baseline.DiscussionOur study evaluated the kidney function of HIV-positive patients who were administered different cART regimens during a 24-month follow-up period. the cART should be started as soon as possible in HIV-infected patients to improve quality of life and reduce the transmission risk ${ }^{1}$. cARTs are highly effective in the virological suppression of HIV infection ${ }^{3}$. Important randomized clinical trials such as START and TEMPRANO have shown that cART achieves approximately a 50\% reduction in morbidi- 
ty and mortality ${ }^{10,11}$. Although it has been shown that early initiation of cARTs reduces the incidence of CKD (by reducing the rates of viral infection, opportunistic infections, immune complex glomerular injury, and HIVAN), it is known that some antiretroviral drugs have the potential to induce nephrotoxicity in persons with normal or impaired eGFR ${ }^{2,6,12}$. Thus, changes in serum creatinine and eGFR during treatment should be correctly interpreted and the treatment should be tailored accordingly. Tenofovir is a nucleotide reverse transcriptase inhibitor, with both TAF and TDF being its pro-drugs. Both of them show similar properties by inhibiting viral replication, which enables them to be used in HIV therapy. TDF, an important component of the first-line ART regimens recommended by the World Health Organization ${ }^{13}$, is recognized as an ART that is most commonly associated with renal adverse reactions. Renal toxicity may occur as a result of tubular dysfunction/injury. Clinical studies performed so far have reported eGFR decline of variable degree within months after starting TDF. As the amount of TDF exposure increases, eGFR decline becomes more pronounced $^{14}$. In a clinical study performed by Patel et al. in western India ${ }^{15}$, kidney dysfunction developed by an average of 150 days after the initiation of TDF therapy. Izzedine et al. ${ }^{16}$ reported that tubular dysfunction or kidney injury occurred approximately 7 months after the start of TDF. In a study of patients that had used TDF for 3 years, eGFR declined by $8 \%$ at the end of 2 years and $11 \%$ at the end of the third year in comparison with the baseline level ${ }^{17}$. In a metanalysis that involved a total of 17 studies, of which 9 were randomized controlled studies, it was concluded that ART regimens involving TDF led to kidney dysfunction more commonly than those that did not involve TDF ${ }^{18}$. Similarly, the D: A:D study reported that 5-year TDF exposure nearly doubled the incidence of CKD, with each additional 1-year exposure to TDF having led to a $23 \%$ increase in the incidence of $\mathrm{CKD}^{19}$. In accordance with the literature reports, we detected a significant eGFR decline after 24 months of therapy in Groups 4 and 5 using regimens containing TDF $(\mathrm{p}<0.001)$ (from 107.03 21.08 to $81.79 \pm 10.4$ and from $106.21 \pm 18.35$ to $80.97 \pm 9.82$, respectively). Clinical studies have shown that kidney injury risk is less with TAF than with TDF ${ }^{20,21}$. The DISCOVER trial, which compared patients receiving Emtricitabine-TAF combination with patients receiving Emtricitabine-TDF combination, showed that creatinine clearance decreased in the TDF group at the end of 48 weeks while it increased in the TAF group ${ }^{22}$. In another study that compared TAF and TDF, although the virological response was more than $90 \%$ in 48 -week EVG/COBI/FTC/TAF and EVG/COBI/FTC/TDF combinations, TAF more favorably affected renal parameters ${ }^{23}$. Although our study detected a slight increase in eGFR at the end of 24 months in Group 3 that contained TAF, this increase was not statistically significant (p:0.886). A comparison of the TDF-containing Groups 4 and 5 and the TAF-containing Group 3 at 12 and 24 months showed a significant increase in serum creatinine level $(\mathrm{p}<0.001)$ and a significant decrease in eGFR $(\mathrm{p}<0.001)$. Another preferred regimen in HIV treatment is the DTG/ABC/3TC combination. This combination can be safely used in patients with end-stage kidney failure ${ }^{24}$. DTG may cause a slight increase in serum creatinine level, depending on its effect on creatinine's tubular secretion. However, this increase does not affect glomerular filtration and renal blood flow ${ }^{25-27}$. Long-term exposure to ABC, another drug used in this combination, has not been linked to increased CKD incidence ${ }^{19}$. In a study of 20 patients receiving DTG/ABC/3TC, only one patient had an increase in serum creatinine level, which was not considered clinically important ${ }^{28}$. The SINGLE study also detected a non-progressive, clinically insignificant increase in serum creatinine level among patients receiving the DTG/ABC/3TC combination ${ }^{29}$. Our study demonstrated a minimal increase in serum creatinine and a slight reduction in eGFR at the end of 24 months with the DTG/ABC/3TC combination. In line with the literature data, however, these changes were not considered statistically significant (p:0.448). Furthermore, we compared eGFR levels of Group 2 with those of the other groups at 12 and 24 months. While no significant difference was observed with Group 3 that received EVG/COBI/FTC/TAF combination, we found significantly lower eGFR levels in Group 4 that received FTC/TDF/DTG treatment and Group 5 that received FTC/TDF/RAL treatment ( $<0.001$ for both comparisons). Our study has several limitations. First, it was a retrospective study; second, it has a small sample size considering the low number of patients in each study group.ConclusionOur study showed that using regimens involving TDF causes renal dysfunction. Therefore, we recommend close monitoring of renal functions, particularly among patients treated with TDF. In our opinion, periodic monitoring of renal function and early diagnosis of potential kidney injury may improve outcomes among HIV-infected patients.

\section{References}


1. Phanuphak N and Gulick RM. HIV treatment and prevention 2019: current standards of care. Curr Opin HIV AIDS 2020 Jan;15(1):4-12.

2. Gameiro J, Jorge S and Lopes JA. HIV and renal disease: a contemporary review. Int J STD AIDS 2018 Jun;29(7):714-719.

3. European AIDS Clinical Society (EACS) GuidelinesVersion 9.1: European AIDS Clinical Society (EACS); 2018. http://www.eacsociety.org/ files/2018_guidelines-9.1-english.pdf. Accessed 13 Jan 2019.

4. Cid-Silva P, Fernandez-Bargiela N, Margusino-Framinan L, et al. Treatment with tenofovir alafenamide fumarate worsens the lipid profile of HIV-infected patients versus treatment with tenofovir disoproxil fumarate, each coformulated with elvitegravir, cobicistat, and emtricitabine. Basic Clin Pharmacol Toxicol. 2019;124(4):479-490.

5. Wyatt CM, Klotman PE. Overview of kidney disease in HIV-positive patients UpToDate,Sep 2020.

6. Ryom L, Mocroft A, Kirk O, et al. Association between antiretroviral exposure and renal impairment among HIV-positive persons with normal baseline renal function: the D:A:D study. J Infect Dis 2013; 207: 1359-69.

7. Mocroft A, Kirk O, Reiss P, et al. Estimated glomerular filtration rate, chronic kidney disease and antiretroviral drug use in HIV-positive patients. AIDS 2010; 24: 1667-78.

8. Scherzer R, Estrella M, Li Y, et al. Association of tenofovir exposure with kidney disease risk in HIV infection. AIDS 2012; 26: 867-75.

9. Alfano G, Cappelli G, Fontana F, et al. Kidney Disease in HIV Infection. J Clin Med. 2019 Aug 19;8(8):1254.

10. Lundgren JD, Babiker AG, Gordin F, et al. INSIGHT START Study Group. Initiation of antiretroviral therapy in early asymptomatic HIV infection. N Engl J Med 2015; 373:795 - 807.

11. Danel C, Moh R, Gabillard D, et al. TEMPRANO ANRS 12136 Study Group. A trial of early antiretrovirals and isoniazid preventive therapy in Africa. N Engl J Med 2015; 373:808 - 822.

12. Milburn J, Jones R and Levy JB. Renal effects of novel antiretroviral drugs. Nephrol Dial Transplant (2017) 32: 434-439.

13. WHO. Updated recommendations on first-line and second-line antiretroviral regimens and postexposure prophylaxis and recommendations on early infant diagnosis of HIV Interim guidance. World Health Organization; 2019 https://www.who.int/hiv/pub/guidelines/ ARV2018update/en/.

14. Tan Q, He YH, Yang TT, et al. Effects of long-term exposure to tenofovir disoproxil fumaratecontaining antiretroviral therapy on renal function in HIV-positive Chinese patients. Journal of Microbiology, Immunology and Infection (2019) 52, 710-719.

15. Patel KK, Patel AK, Ranjan RR, et al. Tenofovir-associated renal dysfunction in clinical practice: an observational cohort from western India. Indian J Sex Transm Dis. 2010;31:30-4.

16. Izzedine H, Isnard-Bagnis C, Hulot J-S, et al. Renal safety of tenofovir in HIV treatment-experienced patients. AIDS. 2004;18:1074-6.

17. Pinto Neto LF, Bassetti BR, Fraga IH, et al. Nephrotoxicity during tenofovir treatment: a three-year follow-up study in a Brazilian reference clinic. Braz J Infect Dis. 2016;20(1):14-18.

18. Cooper RD, Wiebe N, Smith N, et al. Systematic review and meta-analysis: renal safety of Tenofovir Diproxil Fumarate in HIV-infected patients. Clin Infect Dis. 2010;51:496-505.

19. Mocroft A, Lundgren JD, Ross M, et al. for the Data Collection on Adverse events of Anti-HIV Drugs (D:A:D) Study*. Cumulative and current exposure to potentially nephrotoxic antiretrovirals and development of chronic kidney disease in HIV-positive individuals with a normal baseline estimated glomerular filtration rate: a prospective international cohort study. Lancet HIV 2016; 3(1): e23-32.

20. Sax PE, Zolopa A, Brar I, et al. Tenofovir alafenamide vs. tenofovir disoproxil fumarate in single tablet regimens for initial HIV-1 therapy: a randomized phase 2 study. J Acquir Immune Defic Syndr 2014; 67: 52-58.

21. Aloy B, Tazi I, Bagnis CI, et al. Is Tenofovir Alafenamide Safer than Tenofovir Disoproxil Fumarate for the Kidneys? AIDS Rev. Oct-Dec 2016;18(4):184-192.

22. Mayer KH, Molina JM, Thompson MA, et al. Emtricitabine and tenofovir alafenamide vs emtricitabine and tenofovir disoproxil fumarate for HIV pre-exposure prophylaxis (DISCOVER): primary results from 
a randomised, double-blind, multicentre, active-controlled, phase 3, non-inferiority trial. Lancet 2020; 396: 239-54.

23. Sax PE, Wohl D, Yin MT, et al. For the GS-US-292-0104/0111 Study Team*. Tenofovir alafenamide versus tenofovir disoproxil fumarate, coformulated with elvitegravir, cobicistat, and emtricitabine, for initial treatment of HIV-1 infection: two randomised, double-blind, phase 3, non-inferiority trials. Lancet 2015; 385: 2606-15.

24. MS, CA, ME. Abacavir/lamivudine/dolutegravir single tablet regimen in patients with human immunodeficiency virus and end-stage renal disease on hemodialysis. Int J STD AIDS. 2019 Feb;30(2):181-187.

25. Lindeman TA, Duggan JM, Sahloff EG. Evaluation of serum creatinine changes with integrase inhibitor use in human immunodeficiency virus-1 infected adults. Open Forum Infect Dis. 2016;3(2):ofw053.

26. Koteff J, Borland J, Chen S, et al. A Phase 1 study to evaluate the effect of dolutegravir on renal function via measurement of iohexol and para-aminohippurate clearance in healthy subjects. Br J Clin Pharmacol. 2013;75(4):990-6.

27. Raffi. F, Rachlis A, Stellbrink HJ, et al. On behalf of the SPRING-2 study group. Once-daily dolutegravir versus raltegravir in antiretroviral-naive adults with HIV-1 infection: 48 week results from the randomised, double-blind, non-inferiority SPRING-2 study. Lancet 2013; 381: 735-43.

28. Singh RP, Shaik JSB, Skoura N, et al. Effects of Low- and High-Mineral Content Water on the Relative Bioavailability of a Coformulated Abacavir/Dolutegravir/Lamivudine Dispersible Tablet in Healthy Adults. J Acquir Immune Defic Syndr. 2018 Dec 15; 79(5): 631-638.

29. Walmsley S, Baumgarten A, Berenguer J, et al. Brief Report: Dolutegravir Plus Abacavir/Lamivudine for the Treatment of HIV-1 Infection in Antiretroviral Therapy-Naive Patients: Week 96 and Week 144 Results From the SINGLE Randomized Clinical Trial J Acquir Immune Defic Syndr. 2015 Dec $15 ; 70(5): 515-9$.

Table captions

Table 1. Baseline characteristics of patients in the treatment groups

Figure Legends

Figure 1. Flowchart of the enrollment process.

Figure 2. Estimated glomerular filtration rate changes by month in treatment groups and comparison between groups.

Table 1. Baseline characteristics of patients in the treatment groups

\begin{tabular}{|c|c|c|c|c|c|c|}
\hline Parameters & $\begin{array}{l}\text { Group } 1 \\
(n=11)\end{array}$ & $\begin{array}{l}\text { Group } 2 \\
(n=19)\end{array}$ & $\begin{array}{l}\text { Group } 3 \\
(\mathbf{n}=31)\end{array}$ & $\begin{array}{l}\text { Group } 4 \\
(\mathrm{n}=33)\end{array}$ & $\begin{array}{l}\text { Group } 5 \\
(\mathrm{n}=33)\end{array}$ & $\mathbf{p}$ \\
\hline Age & $31.45 \pm 7.91$ & $29.21 \pm 6.91$ & $30.68 \pm 8.47$ & $27.76 \pm 6.71$ & $29.15 \pm 5.62$ & 0.740 \\
\hline $\begin{array}{l}\text { Sex }(\mathrm{Fe}- \\
\text { male/Male) }\end{array}$ & $2 / 9$ & $3 / 16$ & $4 / 27$ & $6 / 27$ & $5 / 28$ & 0.983 \\
\hline BMI & $26.59 \pm 2.14$ & $26.81 \pm 2.46$ & $27.74 \pm 3.98$ & $27.02 \pm 3.73$ & $28.25 \pm 4.16$ & 0.524 \\
\hline HIV-RNA & 0 & $\begin{array}{l}13937.26 \pm \\
24435.15^{\mathrm{e}}\end{array}$ & $\begin{array}{l}2292.10 \pm \\
3020.45^{\mathrm{b}, \mathrm{e}}\end{array}$ & $\begin{array}{l}2208.97 \pm \\
5732.85^{\mathrm{b}, \mathrm{e}}\end{array}$ & $\begin{array}{l}4109.21 \pm \\
12485.02^{\mathrm{b}, \mathrm{e}}\end{array}$ & $<0.001$ \\
\hline CD4 & $\begin{array}{l}549.45 \pm \\
55.21\end{array}$ & $\begin{array}{l}232.74 \pm \\
98.98^{\mathrm{e}}\end{array}$ & $\begin{array}{l}303.29 \pm \\
95.69^{\mathrm{b}, \mathrm{e}}\end{array}$ & $\begin{array}{l}299.58 \pm \\
100.55^{\mathrm{b}, \mathrm{e}}\end{array}$ & $\begin{array}{l}278.24 \pm \\
110.17^{\mathrm{e}}\end{array}$ & $<0.001$ \\
\hline CD8 & $423 \pm 38.30$ & $\begin{array}{l}370.26 \pm \\
74.34\end{array}$ & $\begin{array}{l}423.52 \pm \\
97.77\end{array}$ & $\begin{array}{l}392.85 \pm \\
101.81\end{array}$ & $\begin{array}{l}378.09 \pm \\
102.72\end{array}$ & 0.242 \\
\hline $\begin{array}{l}\text { CD4/CD8 } \\
\text { ratio } \\
\text { Serum } \\
\text { Creatinine } \\
(\mathrm{mg} / \mathrm{dl})\end{array}$ & $1.31 \pm 0.21$ & $0.63 \pm 0.23^{\mathrm{e}}$ & $0.71 \pm 0.14^{\mathrm{e}}$ & $0.75 \pm 0.16^{\mathrm{e}}$ & $0.71 \pm 0.21^{\mathrm{e}}$ & $<0.001$ \\
\hline
\end{tabular}




\begin{tabular}{|c|c|c|c|c|c|c|}
\hline Parameters & $\begin{array}{l}\text { Group } 1 \\
(\mathrm{n}=11)\end{array}$ & $\begin{array}{l}\text { Group } 2 \\
(n=19)\end{array}$ & $\begin{array}{l}\text { Group } 3 \\
(\mathbf{n}=\mathbf{3 1})\end{array}$ & $\begin{array}{l}\text { Group } 4 \\
(\mathrm{n}=33)\end{array}$ & $\begin{array}{l}\text { Group } 5 \\
(\mathrm{n}=33)\end{array}$ & $\mathbf{p}$ \\
\hline Baseline & $0.82 \pm 0.08$ & $0.87 \pm 0.12$ & $0.91 \pm 0.15$ & $0.84 \pm 0.13$ & $0.84 \pm 0.11$ & 0.185 \\
\hline 6 months & $0.84 \pm 0.08$ & $0.89 \pm 0.14$ & $0.88 \pm 0.13$ & $\begin{array}{l}0.95 \pm \\
0.09^{\mathrm{a}, \mathrm{b}, \mathrm{c}}\end{array}$ & $\begin{array}{l}0.96 \pm 0.10 \\
\mathrm{a}, \mathrm{b}, \mathrm{c}\end{array}$ & $<0.001$ \\
\hline 12 months & $0.84 \pm 0.07$ & $0.86 \pm 0.09$ & $0.89 \pm 0.13$ & $\begin{array}{l}1.02 \pm \\
0.08^{\mathrm{e}, \mathrm{f}, \mathrm{g}}\end{array}$ & $\begin{array}{l}0.99 \pm 0.07 \\
\mathrm{e}, \mathrm{f}, \mathrm{g}\end{array}$ & $<0.001$ \\
\hline 24 months & $0.86 \pm 0.08$ & $0.89 \pm 0.06$ & $0.88 \pm 0.11$ & $\begin{array}{l}1.03 \pm 0.05 \\
\mathrm{e}, \mathrm{f}, \mathrm{g}\end{array}$ & $\begin{array}{l}1.04 \pm 0.06 \\
\mathrm{e}, \mathrm{f}, \mathrm{g}\end{array}$ & $<0.001$ \\
\hline \multicolumn{7}{|c|}{$\begin{array}{l}\mathrm{CrCl} \\
\left(\mathrm{mL} / \mathrm{min} / 1.73 \mathrm{~m}^{2}\right)\end{array}$} \\
\hline Baseline & $\begin{array}{l}106.27 \pm \\
16.41\end{array}$ & $\begin{array}{l}104.47 \pm \\
21.99\end{array}$ & $98.13 \pm 22.74$ & $\begin{array}{l}107.03 \pm \\
21.08^{\mathrm{c}}\end{array}$ & $\begin{array}{l}106.21 \pm \\
18.35\end{array}$ & 0.311 \\
\hline 6 months & $\begin{array}{l}104.36 \pm \\
21.04\end{array}$ & $\begin{array}{l}101.26 \pm \\
21.02\end{array}$ & $\begin{array}{l}100.84 \pm \\
15.49\end{array}$ & $\begin{array}{l}89.33 \pm \\
11.60^{\mathrm{a}, \mathrm{b}, \mathrm{c}}\end{array}$ & $\begin{array}{l}89.36 \pm \\
13.25^{\mathrm{a}, \mathrm{c}}\end{array}$ & 0.002 \\
\hline 12 months & $\begin{array}{l}102.82 \pm \\
17.77\end{array}$ & $\begin{array}{l}103.74 \pm \\
16.34\end{array}$ & $99.29 \pm 19.26$ & $\begin{array}{l}83.21 \pm \\
11.81^{\mathrm{a}, \mathrm{f}, \mathrm{c}}\end{array}$ & $\begin{array}{l}85.06 \pm 9.91 \\
\text { a,f,c }\end{array}$ & $<0.001$ \\
\hline 24 months & $98.73 \pm 16.75$ & $98.16 \pm 12.51$ & $99.71 \pm 16.36$ & $\begin{array}{l}81.79 \pm \\
10.43^{\mathrm{a}, \mathrm{f}, \mathrm{g}}\end{array}$ & $\begin{array}{l}80.97 \pm 9.82 \\
\text { a,f,g }\end{array}$ & $<0.001$ \\
\hline
\end{tabular}

$\mathrm{a} ; \mathrm{p}<0.05$ in comparison with group $1 . \mathrm{b} ; \mathrm{p}<0.05$ in comparison with group $2 \mathrm{c} ; \mathrm{p}<0.05$ in comparison with group $3 \mathrm{~d} ; \mathrm{p}<0.05$ in comparison with group $4 \mathrm{e} ; \mathrm{p}<0.001$ in comparison with group $1 \mathrm{f} ; \mathrm{p}<$ 0.001 in comparison with group $2 \mathrm{~g} ; \mathrm{p}<0.001$ in comparison with group $3 \mathrm{~h} ; \mathrm{p}<0.001$ in comparison with group 4

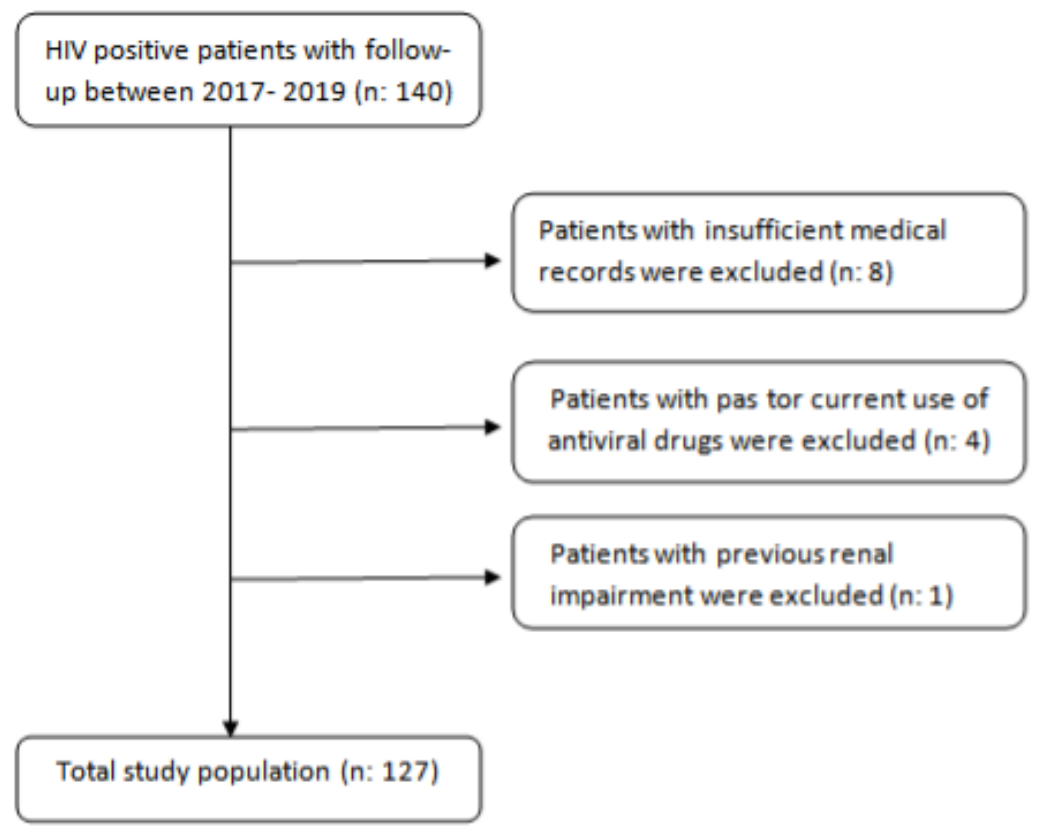




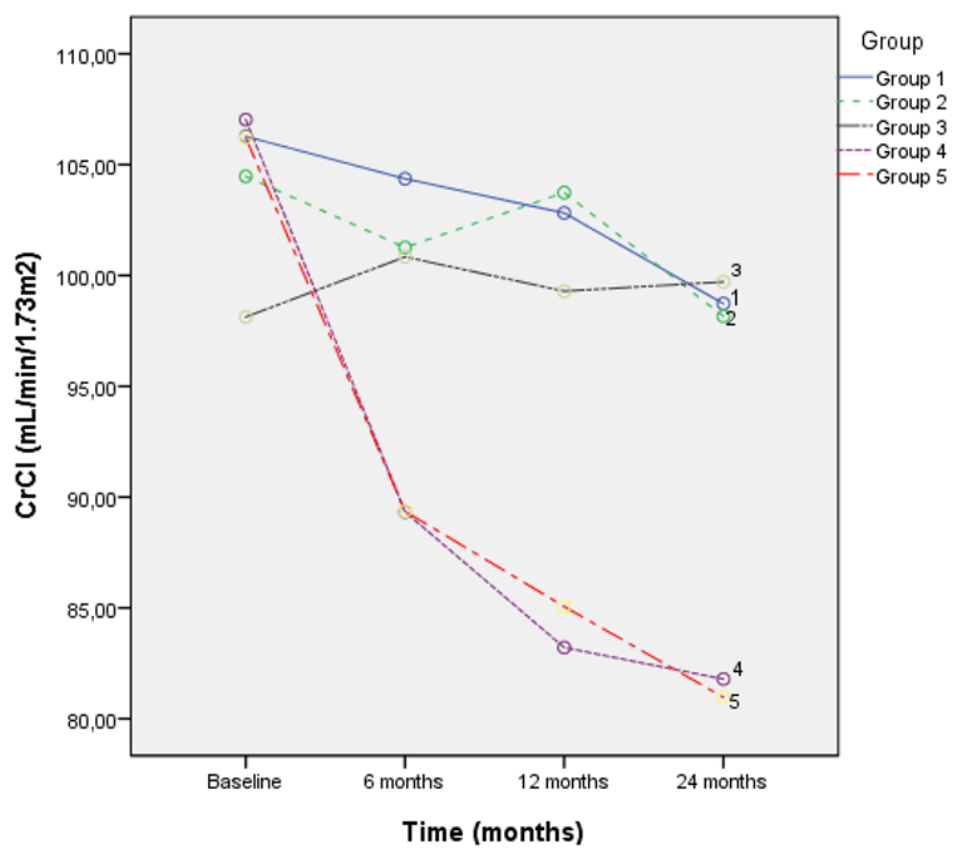

